\title{
G305.20+0.21: A very young class II methanol maser source
}

\author{
Andrew J. Walsh \\ Max-Planck-Institut für Radioastronomie, auf dem Hügel 69, Bonn \\ 53121, Germany.
}

\begin{abstract}
Sensitive radio continuum observations of G305.20+0.21 are performed to look for evidence of an ultracompact HII region. None is found at either 8.64 or $4.8 \mathrm{GHz}$ above a $5 \sigma$ detection limit of about $0.25 \mathrm{mJy}$ in both cases. Since G305.20+0.21 is such a bright infrared source, there is a large discrepancy between the expected radio flux and the observed upper limits. This is interpreted as evidence that G305.20+0.21 is a massive protostar traced by a methanol maser site.
\end{abstract}

\section{Introduction}

Massive star formation (MSF) is an important process to study because massive stars greatly alter their surroundings by way of their powerful winds, dumping energy and momentum into the interstellar medium. Since massive stars typically form in clusters, it is important to study the earliest stages of evolution, before much of the energy and momentum has disrupted the natal environment. Therefore, an important goal of star formation studies is to find an easily identifiable tracer of these early stages.

However, such a tracer is hard to find because massive stars are rare, with few nearby examples to study. Also, their lifetimes are short, so that they evolve quickly on to the main sequence, before they emerge from their natal molecular cloud. This makes them virtually impossible to observe at optical wavelengths.

Traditionally, Class II methanol maser emission has been associated with ultracompact (UC) HII regions (Menten 1991). But in a sample of 233 methanol maser sites, Walsh et al. (1998) found $75 \%$ are not associated with an UCHII region. Furthermore, they showed this cannot be because most methanol maser sites are associated with intermediate mass stars, but they favoured the suggestion that many methanol masers trace a pre-UCHı phase of MSF. If this is the case, then $6.7 \mathrm{GHz}$ methanol maser emission (the strongest class II methanol maser) may be an easily identifiable tracer of the early stages of MSF.

Walsh et al. (2001, MNRAS, in press) have provided encouraging evidence for this in a sample of mid-infrared sources associated with methanol maser sites. Such sources have bolometric luminosities consistent with massive stars, but many show no evidence for radio continuum emission from an UCHII region. One of the most luminous sources in this survey is G305.20+0.21, but it is not associated with any known UCHII region. In this paper, I report on very sensitive observations to look for weak radio continuum from this source. 


\section{Observations}

The observations were performed on the 14th of February, 2000 with the Australia Telescope Compact Array. The observing frequencies were $8.64 \mathrm{GHz}$ and $4.80 \mathrm{GHz}$, both observed with $128 \mathrm{MHz}$ bandwidth. The configuration used was $6 \mathrm{~A}$, but only 5 of the 6 antennas were available for the observations. The observations lasted for 12 hours, to most fully sample the UV plane, and to obtain a high sensitivity. 1934-638 was observed once and was used as the primary calibrator. 1352-63 was observed at intervals of 20 minutes throughout the observing period, and was used as a secondary calibrator. Data reduction was performed using MIRIAD, using standard techniques.

\section{Results}

The $4.8 \mathrm{GHz}$ radio continuum is shown as contours overlayed on the $\mathrm{K}$-band $(2.2 \mu \mathrm{m})$ greyscale image of Walsh et al. (1999) in Figure 1. The absolute positional accuracy of the radio continuum data is $1^{\prime \prime}$, and that of the K-band image is $2^{\prime \prime}$. Thus, the registration between the coordinate frames should be no worse than $3^{\prime \prime}$. However, a comparison of the $4.8 \mathrm{GHz}$ contours and $\mathrm{K}$-band greyscale images shows three features that can be matched for better registration:

1. The cometary HiI region seen centred around $13118.6,-623442$ is coincident with a near-infrared (NIR) star. At the position of the star, the radio continuum contours show a dip in the emission. This dip implies the NIR star is physically connected to the HII region, and is possibly its powering source. Thus, the dip in the radio continuum emission is likely to be a result of shielding by the stellar wind of the star.

2. A second cometary HII region is located at $1311 \quad 17.8,-623459$. A NIR star is also found at this position, with the radio continuum contours similarly dipping at the position of the star.

3. A weak, unresolved radio continuum source is found at $131110.0,-6234$ 40. This is coincident with a NIR star.

The two methanol maser sites located in this field (Norris et al. 1993) are shown by the white (G305.20+0.21) and black (G305.21+0.21) crosses. Neither is associated with any detected radio continuum emission, with $5 \sigma$ upper limits given in table 1.

\begin{tabular}{ccc}
\hline & G305.20+0.21 & G305.21+0.21 \\
\hline $4.80 \mathrm{GHz}$ & $0.27 \mathrm{mJy}$ & $0.24 \mathrm{mJy}$ \\
$8.64 \mathrm{GHz}$ & $0.24 \mathrm{mJy}$ & $0.15 \mathrm{mJy}$ \\
\hline \hline
\end{tabular}

Table 1. $5 \sigma$ upper limits on the non-detection of radio continuum emission for the position of each maser site at both observing frequencies. 


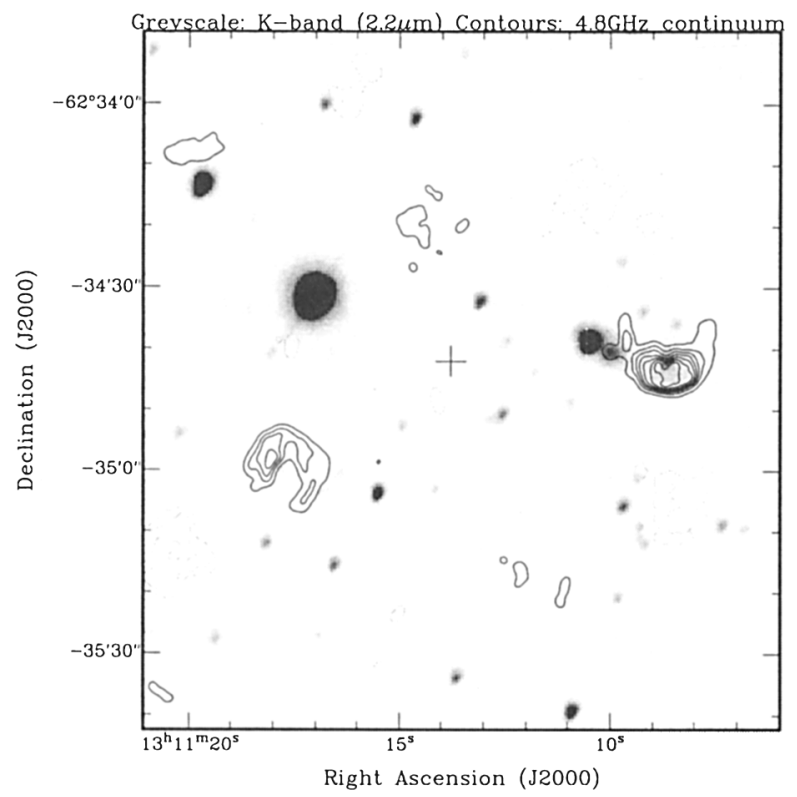

Figure 1. Greyscale is $\mathrm{K}$-band $2.2 \mu \mathrm{m}$ emission and contours are $4.8 \mathrm{GHz}$ radio continuum emission. The two methanol maser sites are located at the centre of the two crosses.

\section{Discussion}

\subsection{G305.20+0.21}

This maser site, shown as the white cross in Figure 1, is not associated with any detected radio continuum emission. However, there is a bright NIR source at this position. A comparison of its NIR magnitudes $(\mathrm{H}=9.0 \mathrm{~m} ; \mathrm{K}=7.0 \mathrm{~m} ; \mathrm{L}=3.6 \mathrm{~m})$ indicates it is the reddest, and therefore the most deeply embedded, object detected in the field. The bolometric luminosity of this source is determined to be $2.6 \times 10^{5} \mathrm{~L}_{\odot}$ (Walsh et al. 1997), assuming a distance of $4.5 \mathrm{kpc}$. A single ZAMS star with this bolometric luminosity has a spectral type of O5.5 (Panagia 1973). Such and O5.5 star will produce an UCHII region, with a flux that can be derived from equation 3 of Kurtz, Churchwell, \& Wood (1994). In the case of G305.20+0.21, a flux of $770 \mathrm{mJy}$ is expected at $8.64 \mathrm{GHz}$. The $5 \sigma$ upper limit lies more than 3 orders of magnitude below this expected level. What could be the reason why there is such a large discrepancy between the expected and observed (lack of) radio continuum emission?

- A star cluster is responsible for the observed emission, rather than a single star: A star cluster with a similar bolometric luminosity will have a smaller radio flux than a single star. Using the modelled IMFs of Walsh et al. (2001, MNRAS, in press), the most likely range for the radio flux of a cluster, with bolometric luminosity $2.6 \times 10^{5} \mathrm{~L}_{\odot}$, is between $108 \mathrm{mJy}$ and $770 \mathrm{mJy}$. The lower limit $(108 \mathrm{mJy})$ is still nearly 3 orders of magnitude above the detection limit. 
- Dust absorbs much of the UV radiation within the UCHII region, thereby reducing the radio flux. However, Molinari et al. (2000) have studied the dust properties of a sample of similar objects. They conclude that dust is not a dominant factor in determining the radio flux of UCHII regions. Thus, it is unlikely that the 3 orders of magnitude discrepancy seen in this source can be accounted for by dust.

- The UCHII region is kept so small that it cannot be detected. This may occur because there is a high density of material surrounding the UCHII region. However, Forster \& Caswell (2000) show that it is unlikely an UCHII region can be kept so small for times equivalent to the maser life time $\left(\sim 10^{5}\right.$ years $)$.

Therefore, the most likely interpretation for G305.20+0.21 is that it is at such an early stage of evolution that the UCHII region has not had time to develop yet. Since massive stars do not have a pre-main sequence stage, it is likely $\mathrm{G} 305.20+0.21$ is a massive protostar.

\section{References}

Forster, J. R., \& Caswell, J. L., 2000, ApJ, 530, 371

Kurtz, S., Churchwell, E., \& Wood, D. O. S., 1994, ApJS, 91, 659

Menten, K. M., 1991, ApJ, 380, L75

Molinari, S., Brand, J., Cesaroni, R., \& Palla, F., 2000, A\&A, 355, 617

Norris, R. P., Whiteoak, J. B., Caswell, J. L., Wieringa, M. H., \& Gough, R. G., 1993, ApJ, 412, 222

Panagia, N., 1973, AJ, 78, 929

Walsh, A. J., Hyland, A. R., Robinson, G., \& Burton, M. G., 1997, MNRAS, 291,261

Walsh, A. J., Burton, M. G., Hyland, A. R., \& Robinson, G., 1998, MNRAS, 301,640

Walsh, A. J., Burton, M. G., Hyland, A. R., \& Robinson, G., 1999, MNRAS, 309,905 